\title{
Characteristics of co-dependence among wives of persons with substance use disorder in Iran
}

\begin{abstract}
Substance use disorder is known to adversely affect both the user as well as his or her family members. A consequence of chemical dependence on family members is co-dependence. Codependence is associated with an excessive focus on the needs and behaviors of the person with substance use disorder. Most studies in the field of drug-related problems in Iran have focused on the substance user, while few studies have been carried out on the family members. A qualitative case study approach was employed to explore characteristics of codependence among wives of persons with substance use disorder in Iran where substance use disorder is a notable social harm. Purposive snowball sampling technique was used to identify 11 Iranian wives of individuals with substance use disorder. The data were collected through in-depth, face-to-face interviews, non-participation observation, and document analysis. Data were analyzed using the constant comparative method. The analysis yielded five main characteristics of co-dependence among wives of persons with substance use disorder, namely: Denial, enabling behavior, low self-worth, enmeshed self, and weak spiritual relationship with God. The findings showed that except for a weak spiritual relationship with God, most of the characteristics of co-dependence among the participants were similar to the traits of co-dependents found in previous studies conducted outside Iran.
\end{abstract}

Keyword: Co-dependence; Wife of a person with substance use disorder; Substance use disorder; Qualitative case study 